\title{
Tension Gastrothorax with Gastric Perforation Secondary to Bochdalek Hernia in an Adult, Case Report
}

\author{
Tolentino Gonzalez Christian S. MD ${ }^{1}$, Christian Omar Ramírez Serrano Torres MD ${ }^{1}$, Diana \\ Patricia Jiménez Carranza MD ${ }^{1}$, Ricardo Daniel Romero Morelos MD $^{2}$, Iván Hernández \\ Altamirano $\mathrm{MD}^{3}$, Marco Antonio Romero López $\mathrm{MD}^{3}$, Javier Omar Valencia Jiménez $\mathrm{MD}^{4}$, \\ Lorenzo Rojas Domínguez MD $^{5}$, José Trinidad Ibarra Ortega MD $^{1}$, Javier Omar Valencia \\ Jiménez $\mathrm{MD}^{4}$, Moises Alejandro Perdomo Galvan $\mathrm{MD}^{3}$, Medina Andrade Luis Angel MD ${ }^{6 *}$ \\ ${ }^{1}$ General Surgery Department, Gneral Zone Hospital \#47 “Vicente Guerrero”, Mexican Institute of Social \\ Security, México City \\ ${ }^{2}$ Resident of General Surgery, Angeles del Pedregal Hospital, Mexico City \\ ${ }^{3}$ General Surgery Department, Dario Fernandez Fierro General Hospital, Institute of Security and Social \\ Services for the State Workers, Mexico City \\ ${ }^{4}$ General Surgery Department, Star Medica Hospital Center, Mexico City \\ ${ }^{5}$ General Surgery Department, San Rafael Hospital, México City \\ ${ }^{6}$ General Surgery Department, General Zone Hospital $1^{a}$ Dr. Rodolofo de Mucha Macias, Mexico City
}

*Corresponding Author: Luis Angel Medina Andrade, General Surgeon, General Surgery Department, General Zone Hospital $1^{a}$ Dr. Rodolofo de Mucha Macias, Mexico City. Email: medicaleditions@gmail.com

\section{INTRODUCTION}

Bochdalek hernia was described in the literature since 1847 by the Anatomist Vincent Alexander Bochdalek, and described as an inappropriate fusion of the transverse septum and the pleuroperitoneal fascia ${ }^{3}$. This pathology is frequently diagnosed after birth or in the first 48 hours of life, and is characterized by dyspnea, tachypnea, thoracic retractions, pallor and cyanosis, and all this signs related to lung hypoplasia by the occupation of thoracic cavity by abdominal organs. When it is suspected an urgent surgery is required to allow patient to survive.

This hernia defect is localized in the left posterolateral diaphragm, with 80 to $90 \%$ of the cases in this side, and secondary to a failure in the closure during embryogenesis between 7 to 10 weeks of gestation ${ }^{3,4}$.

The incidence of congenital diaphragmatic hernias is about 1 case per 12500 live births, and is associated with a mortality of $50 \%$ in neonates ${ }^{5,6}$. The presentation in adult life is very rare, with an incidence between the diaphragmatic hernias of $0.17-6 \%$, with only about 130 cases reported in the literature ${ }^{1}$

\section{Case Presentation}

This was a female patient presenting to emergency department complaining for a two days of evolution abdominal and retrosternal pain, associated with difficulty to amplexion and amplexation, with an intensity of 10/10 in the visual analogue scale of pain. She referred previous episodes of epigastric pain, vomiting, nausea and constipation since 8 years old, other pathological background was denied. Two days before, she had the same symptoms and was treated with analgesics with minimal improvement. In this chance the pain did not respond to analgesics so laboratory test and $\mathrm{x}$ rays were requested reporting: glucose 203.5 $\mathrm{mg} / \mathrm{dl}$, leucocytes $12.6 \times 10^{3} \mathrm{~mm}^{3}$, hemoglobin $18.4 \mathrm{~g} / \mathrm{dL}$, platelets: $274.0 \mathrm{x} 10^{3} / \mathrm{mm}^{3}$, prothrombin time: 25.0 seconds, INR: 2.09, thromboplastin partial time: 37.8 seconds. Chest $\mathrm{X}$ ray with a bubble image in left hemithorax and an air-liquid level displacing the cardiac silhouette and trachea to the right, right sub diaphragmatic air (Figure 1).

At physical exam with 15 points in Glasgow scale, referring intense abdominal and thoracic pain, with respiratory insufficiency, arterial tension of $85 / 60 \mathrm{mmHg}$, cardiac rate of 145 
beats per minute, respiratory frequency of 18 breaths per minute, temperature of $36^{\circ} \mathrm{C}$, respiratory distress signs, bowel sounds in left hemithorax, abdomen with epigastric pain at palpation and diminished bowel sounds.

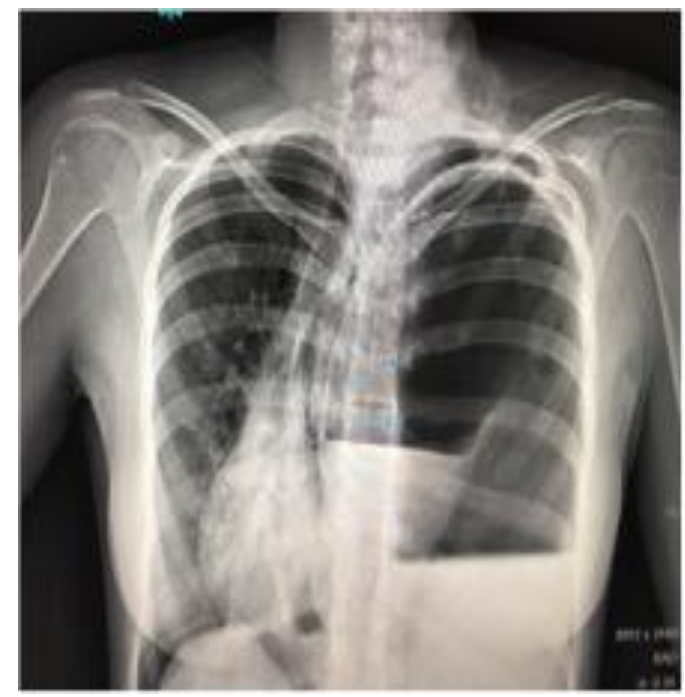

Figure1. Chest $x$-ray with gastric bubble in left thorax and the classical air-liquid level

A diaphragmatic hernia with intestinal perforation was suspected and an urgent laparotomy scheduled immediately, with no TAC requested by the actual septic shock. A left posterolateral diaphragmatic defect of $10 \mathrm{~cm}$ was found, with stomach, colon and spleen inside left hemithorax and intestinal liquid in this location (Figure 2). The abdominal structures were reduced to the abdominal cavity, and a 2 $\mathrm{cm}$ perforation in the stomach near the His angle identified for a primary closure in two planes, the thorax washed with saline solution and a diaphragmatic closure with prolene 00 continuous stitch was completed. A jejunostomy was performed at $40 \mathrm{~cm}$ of the Treitz ligament and a thoracic tube placed in left hemithorax.

The patient stays in the Intensive Care Unit for 48 hours, and a thoracic tube was placed in the right hemithorax by tension pneumothorax. After feeding by jejunostomy was tolerated the oral feeding was introduced without problems and paint was discharged.

She returns after 7 days complaining of fever $39^{\circ} \mathrm{C}$ and tachycardia. A CT scan was performed showing a residual left thoracic abscess that was resolved by guided punction and Malecot drain placement, removed 7 days after and treated with double antibiotic. She was discharged and in the follow up at 15 days the $\mathrm{x}$ ray shows the left hemithorax without pathology (Figure 3).
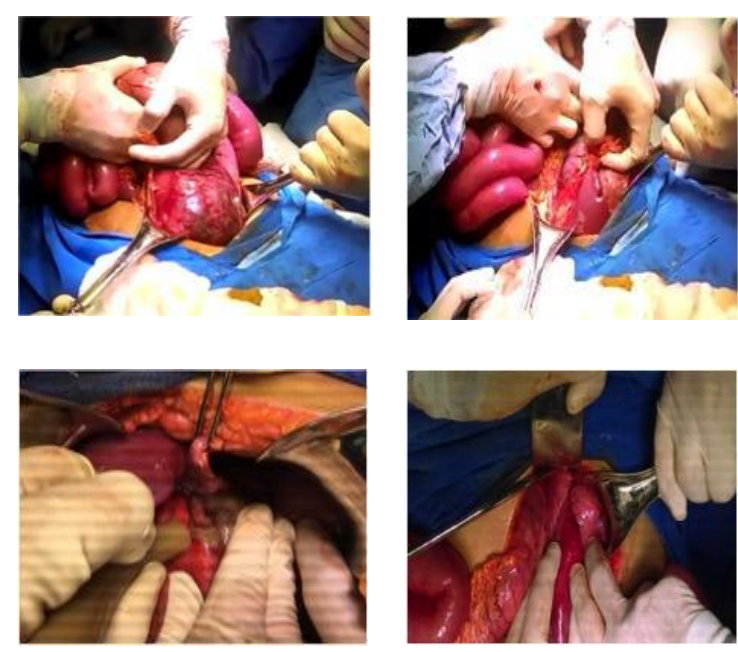

Figure2. A. Stomach reduced to abdominal cavity with ischemia signs in the wall. B. Spleen reduced to abdomen. C. Gastric perforation near the His angle. D. Gastric perforation repair.

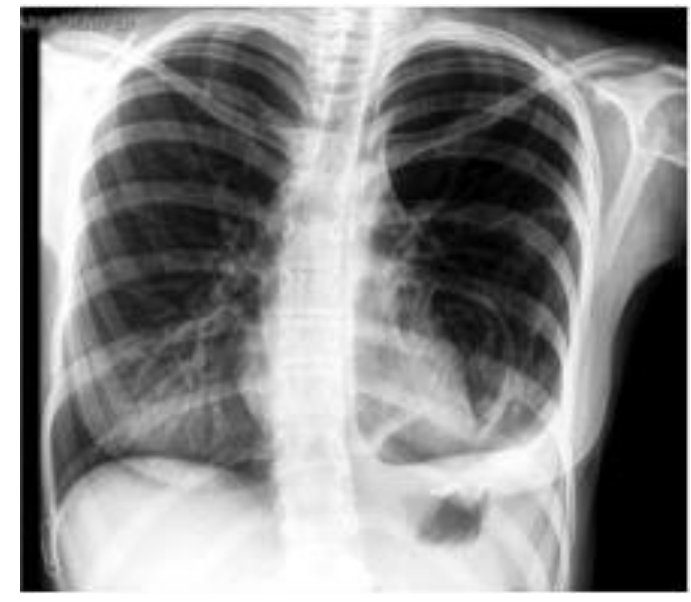

Figure3. Control chest $x$-ray after diaphragmatic repair and 2 weeks after residual abscess drainage.

\section{DisCUSSION}

Congenital diaphragmatic hernias are very rare in the adulthood. As mentioned before, the presentation uses to be after birth, with respiratory distress as principal symptom and need an urgent surgery, with a mortality about $50 \%$.

The bochdalek hernia constitutes one of the commonest congenital hernias, with about 78$90 \%$ of the cases, and is a left posterolateral diaphragmatic defect. It is most common in females $(77 \%)$ and $5 \%$ are presented in the adulthood. In the last, the diaphragmatic hernia is associated with epigastric or retroesternal pain, sometimes with caught or a momentary respiratory distress that lasts some seconds to minutes, nausea, vomiting or occasional constipation. Unfortunately, the correct diagnosis usually is stablished during a gastrointestinal emergency, with a mortality of 
$30 \%$. ${ }^{8}$ In fact, in adults there is no more than 140 cases reported ${ }^{9,10}$.

It was accepted that the left hernias were more common than the right ones, but a study of Mullins et. al. demonstrated that $68 \%$ are right sided, but there are under diagnosed because the close of the defect by the liver make them asymptomatic ${ }^{11}$.

The main organs involved in herniation are stomach, small bowel, colon and spleen. The herniation of this organ could constitute an emergency like in the present case with the presence of gastrothorax. In this cases after a couple of hours the blood supply can be interrupted and the tissue become necrotic with the subsequent perforation, sepsis and septic shock if this is not resolved properly.

To confirm the diagnosis the clinical history is basic, for the mentioned chronic symptoms. At physical examination the bowel sound in thorax are characteristic, with or without retroesternal or epigastric pain. Brown et. al. in their review of the literature reported that only $14 \%$ present a symptom during physical exam. The more common symptoms were chest pain (69\%), intestinal obstruction (39\%), respiratory distress $(37 \%)$, strangulation (28\%), dysphagia (3\%), bleeding $(4 \%)$ and other $(9 \%)^{12}$.

In case of gastrointestinal perforation acute abdomen signs and sepsis could be found. To confirm the diagnose the first study is the chest and abdominal x-ray, in which we could appreciate the left thorax occupied by small bowel or the gastric bubble with an air-liquid level, this hemi diaphragm could be elevated and sometimes with pleural effusion. The gold standard for diagnosis is the CT scan, which could help us to determine the organs involved, if there is perforation or bleeding, and one of the more important characteristics: the size of the defect. This last one is important to determine the approach and the technique to repair the defect ${ }^{13}$.

In cases in which perforation is suspected like in the urgent repairs the most appropriate approach is the abdominal, because independently of the organ involved it could be fixed this way, and the thoracic approach must represent a limited approach that do not allow us to repair most of the complications. If there is no suspected perforation and the diagnosis is made in the course of a routine exam and the surgery is scheduled, the thoracic or abdominal approaches are feasible, with the minimally invasive techniques as safe ones to complete this task. In cases of a diaphragmatic hernia of less than 10 $\mathrm{cm}$ the direct closure by suture with interrupted stitches with a non-absorbable suture is recommended. In the laparoscopic approach this closure must be very difficult for the high tension to apply in the stitches and for this reasons the mesh must have a side with a biological cover or a polytetrafluoroethylene cover to allow the contact with bowel and avoid the risk of perforation and gastrointestinal fistula after repair ${ }^{14}$.

According to the clinical presentation the prognostic could be estimated. In the case of an elective surgery the mortality is about 3\%, increasing to $32 \%$ in an emergency scenario like visceral perforation, necrosis or intestinal obstruction $^{6}$. In the present case the open approach was decided for the gastrointestinal compromise with the possibility of perforation by the sepsis signs, and the high mortality encourage us to the postsurgical management by the intensive care unit, with an APACHE II score of 25 points and a calculated mortality of $53.3 \%$ at admission, but with an excellent evolution and limited complications that allowed good results at the end.

\section{CONCLUSiON}

The diaphragmatic defects in adults are very rare, but must be considered in patients with thoracic pain and concomitant abdominal symptomatology that most of the times are presented as chronic symptoms that were not correctly studied. The implications of misdiagnosis are the complications associated with this pathology that increase the mortality in a significant amount in the course of an acute setting, and limit the surgical options like the minimally invasive approach to bring the patient the better results.

\section{REFERENCES}

[1] Schumacher L, Gilbert S. Congenital diaphragmatic hernia in the adult. Thorac Surg Clin 2009; 19:469-472.

[2] Chai Y, Zhang G, Shen G. Adult Bochdalek hernia com $\neg$ plicated with a perforated colon. J Thorac Cardiovasc Surg. 2005; 130:1729-30.

[3] Allison Gilbert, MD Tension gastrothorax as a complication of bochdalek hernia, The Journal of Emergency Medicine, Vol. 52, No. 1, pp. e17-e18, 2017.

[4] Harrington DK, Curran FT, Morgan I, Yiu P (2008) Congenital Bochdalek hernia presenting with acute pancreatitis in an adult. J Thorac Cardiovasc Surg 135(6): 1396-1397. 
[5] Laaksonen E, Silvasti S Hakala T. Right-sided Bochdalek hernia in an adult: a case report. J Med Case Rep 2009; 3:9291.

[6] Medina Andrade Luis Angel, Medina Andrade Laura G., Medina Andrade Abraham Alejandro, Ortiz Ramirez Grecia,et. al. International Journal of Surgery Case Reports 5 (2014) 743-745

[7] Medina Andrade Luis Angel, Serrano Collazos S., Martinez Ferretiz M., Perez Muñoz F., et. al. Bochdalek hernia with Gastric Necrosis in a 54year-old man, Case Report and Review of the Literature. MOJ Clin Med Case Rep 2016, 4(2): 00084

[8] Deb SJ. Massive right-sided Bochdalek hernia with two unusual findings: a case report. J Med Case Rep 2011; 5:519.

[9] T. Toydemir, H. Akinci, M. Tekinel, E. Süleyman, B. Acunas, M.A. Yerdel, Laparoscopic repair of an incarcerated Bochdalek hernia in an elderly man, Clin. (Sao Paulo) 67 (2) (2012) 199e201.
[10] Mullins ME, Sanjay JS, Saini S, Mueller PR. Prevalence of incidental Bochdalek's hernia in a large adult population. AJR 2001; 177:363366.

[11] S.R. Brown, J.D. Horton, E. Trivette, L.J. Hofmann, J.M. Johnson, Bochdalek hernia in the adult: demographics, presentation, and surgical management, Hernia 15 (1) (2011 Feb) $23 \mathrm{e} 30$.

[12] Esmer D, Alvarez Tostado J, Alfaro A, Carmona R, Salas M. Thoracoscopic and laparoscopic repair of complicated Bochdalek hernia in adult. Hernia

[13] Dalencourt G, Katlic MR. Abdominal compartment syndrome after late repair of Bochdalek hernia. Ann Thorac Surg 2006; 82:721-722.

[14] Rout S, Foo FJ, Hayden JD, Guthrie A, Smith AM. Right-sided Bochdalek hernia obstructing in an adult case report an review of the literature. Hernia 2007; 11:359-62

Citation: Tolentino Gonzalez Christian S., Christian Omar Ramírez Serrano Torres, Diana Patricia Jiménez Carranza, Ricardo Daniel Romero Morelos, Iván Hernández Altamirano, Marco Antonio Romero López et al. Tension Gastrothorax with Gastric Perforation Secondary to Bochdalek Hernia in an Adult, Case Report. ARC Journal of Surgery. 2017; 3(4): 1-4. doi: dx.doi.org/10.20431/2455-572X. 0304001.

Copyright: (C) 2017 Authors. This is an open-access article distributed under the terms of the Creative Commons Attribution License, which permits unrestricted use, distribution, and reproduction in any medium, provided the original author and source are credited. 